\title{
DESIGN AND DEVELOPMENT OF SAPLING MONITORING SYSTEM
}

\author{
Ramandeep Kaur $^{1}$ and Balwinder Singh ${ }^{2}$ \\ 1-2 Acadmic and Consultancy Services Division, \\ Centre for Development of Advanced Computing(C-DAC), Mohali, India
}

\begin{abstract}
The measurement of a plant growth without interrupting its natural growth is essential since its diameter and height are related to the development of a tree as well as the water contents. Therefore main objective of this project is to find the diameter and height of a plant. Hence in this proposed work, an interface between a C3008 smart camera and a computer has been developed. Main advantage of this camera module is that it has digital output and digital video port that supplies a continuous 8-16 bit range of data stream. It is used to capture the image of a plant at different intervals of time. A MATLAB GUI has been developed for performing Sobel Edge Detection on a plant image to determine its height, maximum and minimum width. Such programs enable us to automatically measure and record the various parameters of a plant. The results attained prove that the system is capable of measuring the changes (diameter and height changes) of plant's growth accurately.
\end{abstract}

\section{KEYWORDS}

Stem diameter and height, Sobel operator, edge detection.

\section{NEED AND SIGNIFICANCE OF PROPOSED PROJECT}

Growth of plant is influenced by environmental conditions and topography. There is a need to measure dynamics of plant to understand how plants respond to variations in water contents and long term growth conditions. Many studies have found that the variation of the diameter and height of plant stem has a prominent correlation ship with water status, temperature, light and humidity.

Measuring the growth of plant using strain gauge does not provide accurate values of plant's growth. It required large precision. The output signal is weak and unwanted signal and interference is also introduced. Thermal effect also arises in strain gauge method. In the monitoring system using LVDT sensors in order to acquire the stem diameter has also some severe shortages such as that we need to change sensors of different scale to fit the different range of diameters. We can only get the increment of the diameter while leaving the real value of the stem diameter unknown to us. The sensor may restrain the natural growth of the crops.

Our device can not only acquire the real-time diameter of stem but also measure height and its increment, and also work without interrupting the natural growth of the crop. Output values accurate and precised. Also there is continuous analog video signal for the uninterrupted monitoring of plant every time. No storage device is required . 


\section{INTRODUCTION}

Growth of plant is influenced by environmental conditions and topography. There is a need to measure dynamics of plant to understand how plants respond to variations in water contents and long term growth conditions. Many studies have found that the variation of the diameter and height of plant stem has a prominent correlationship with water status, temperature, light and humidity. Evaluation of these aspects by variation of plant stem is suitable for automatic monitoring in a long period of time. Thus, due to foresaid factors automatic irrigation approach is of utmost importance these days.

The objective of this project is to find the diameter and height of a plant. This project consists of two parts which can be divided into hardware and software. The basic structure consists of digicam module, controller and PC. The system comes into an action when a user presses the button, mounted on it for the user interface. In first module, there is camera interfaced to AVR controller to acquire an image. Main advantage of this camera is that it has digital output. Digital port that supplies a continuous $8-16$ bit wide range data stream. It will capture the image of plant at different interval of time. Digicam pass the data to the PC through USB. All the functions of camera contrast, brightness, intensity etc. are programmable through $\mathrm{I} 2 \mathrm{C}$ interface. For further processing of the image, it is fed to the PC. In this module image is detected, edge detection, data acquisition and storage of data is performed. Motor is used to move the camera on belt to capture images of different plants.

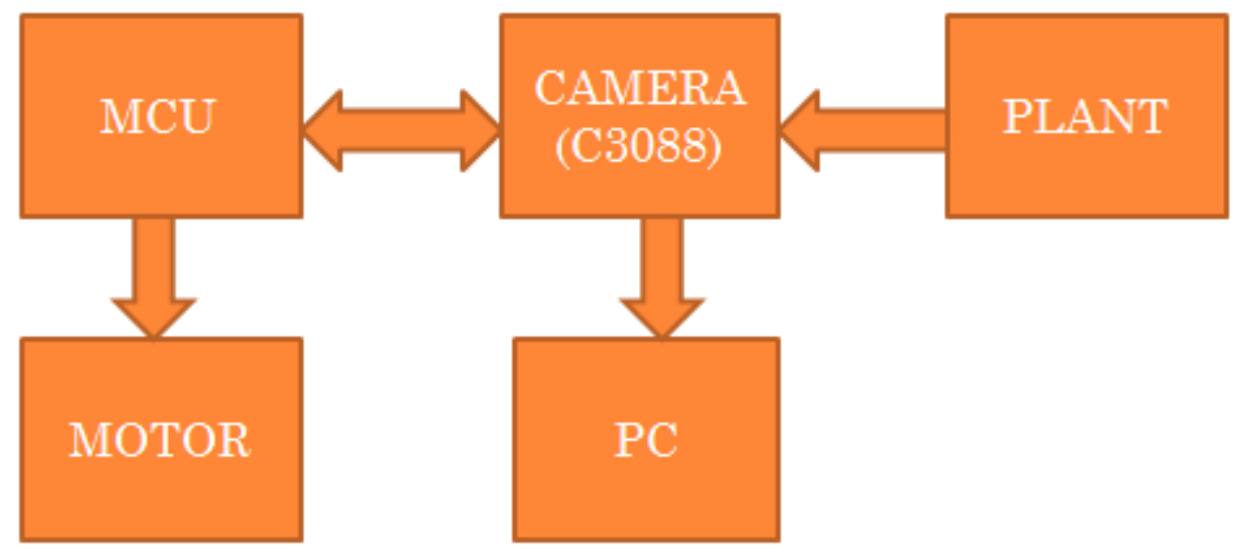

Figure 1. The block diagram system

\section{RELATED WORK}

Currently, there are various techniques for measuring stem diameter and height. Several experiments were conducted to investigate the development and expansion of a plant. The results of these experiments indicate that the variation of the diameter of plant stem, height, temperature and humidity has a prominent correlationship with the water status. The conventional monitoring system used LVDT sensors in order to acquire the stem diameter has some severe shortages such as that we need to change sensors to different scale to fit the different range of diameters. We can only get the increment of the diameter while leaving the real value of the stem diameter unknown to us. N.M Khairi et. al. [1] measures the stem changes using strain gauge. Strain gauges are sensors that rely on the change in gauge resistance in determining the strain within the gauge section. Measuring the growth of plant using strain gauge does not provide accurate values of 
plant's growth. It required large precision. The output signal is weak and unwanted signal and interference is also introduced. Thermal effects also arises in strain gauge method. Both of the above discussed method may also restrain the natural growth of the crops. Jiaxing Che et. al. [2] developed a model using computer vision and embedded systems. it was based on smart camera, computer vision technology and embedded DSP processor technology. Ariff et. al. [3] had measured Stem diameter using a digital caliper, with the readings taken midway on the stem. Heights of the samples were measured using a measurement tape, from the tip of the shoot to the ground.

\section{THE ALGORITHM USED FOR SAPLING MONITORING SYSTEM}

In digital image, the so-called edge is a collection of the pixels whose gray value has a step and it also refers to the fraction where the brightness of the image local area changes considerably. The gray profile in this section can normally be seen as a step. In a small buffer area, a gray value quickly changes to an additional whose gray value is mainly different with it. Edge broadly exists between objects and backgrounds and between objects and primitives. Therefore, the general method of edge detection is to study the changes of a single image pixel in a gray area, use the dissimilarity of the edge neighboring firstorder or second-order to detect the edge. Edge detection is primarily the dimension, detection and position of the changes in gray image. Image edge is the most basic features of the image. When we examine the objects, the transparent part we see firstly is edge and line. According to the structure of the edge and line, we can know the object organization. Edge extraction is an significant technique in graphics processing and feature extraction. First of all, use edge enhancement operator to highlight the local edge of the image. Then, define the pixel "edge strength" and set the threshold to extract the edge point. However, because of the noise and the blurring image, the edge detected may not be constant. There are various edge detection algorithms available. In this proposed work, Sobel Edge Detection algorithm has been used.

\subsection{Sobel edge detection}

The Sobel operator performs a 2-D spatial gradient measurement on an image and emphasizes regions of high spatial gradient that correspond to edges. In general it is used to find the approximate absolute gradient magnitude at each point in an input greyscale image. Compared to other edge operator, Sobel has two main advantages:

1. Since the introduction of the average factor, it has some smoothing effect to the random noise of the image.

2. Because it is the differential of two rows or two columns, so the elements of the edge on both sides has been enhanced, so that the edge seems thick and bright.

The operator consists of a pair of $3 \times 3$ convolution kernels as shown in Figure 2. Single kernel is simply the other rotated by $90^{\circ}$. 


\begin{tabular}{|c|c|c|}
\hline-1 & 0 & +1 \\
\hline-2 & 0 & +2 \\
\hline-1 & 0 & +3 \\
\hline
\end{tabular}

\begin{tabular}{|c|c|c|}
\hline+1 & +2 & +1 \\
\hline 0 & 0 & 0 \\
\hline-1 & -2 & -1 \\
\hline
\end{tabular}

Figure 2. Masks used by Sobel Operator

These kernels are designed to respond maximally to edges running vertically and horizontally relative to the pixel grid, single kernel for each of the two perpendicular directions. The kernels can be applied separately to the given image, to produce separate measurements of the gradient component in each orientation (call these $G x$ and $G y$ ). These can then be joint together to find the absolute magnitude of the gradient at each point and the orientation of that gradient . The gradient magnitude is given by [4]:

$$
|G|=\sqrt{\mathrm{Gx}^{2}+\mathrm{Gy}^{2}}
$$

Although typically, an approximate magnitude is computed using:

$$
|G|=|G x|+|G y|
$$

which is much faster to compute.

\section{RESULTS AND DISCUSSIONS}

A matlab GUI has been developed for performing Sobel Edge Detection on a plant image to determine its height and width. Figure 3 shows the two different options for the selection of a plant image. That is either we can manually cropped the image (pressing 2) or select the full image (pressing 1).

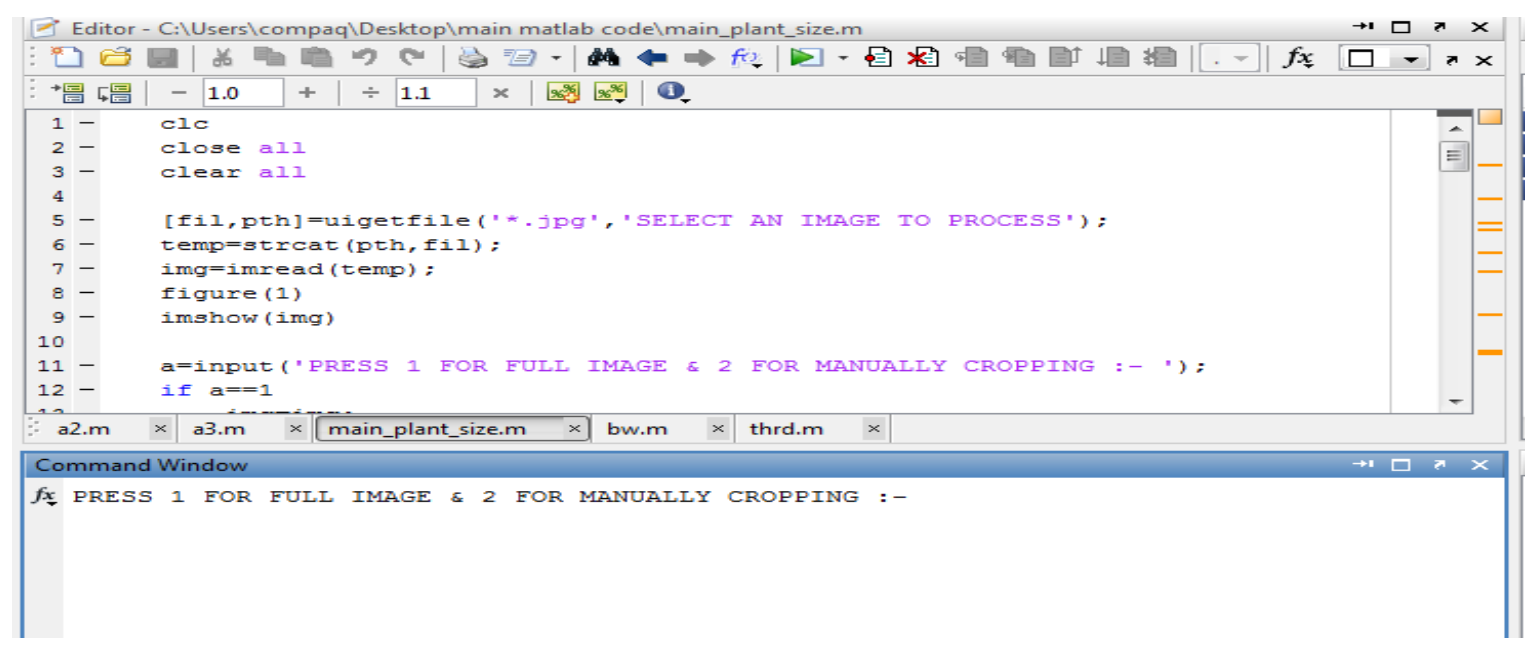

Figure 3. Image selection criteria

Figure 4 shows the selection of plant image with provision of cropping by pressing 2 . 


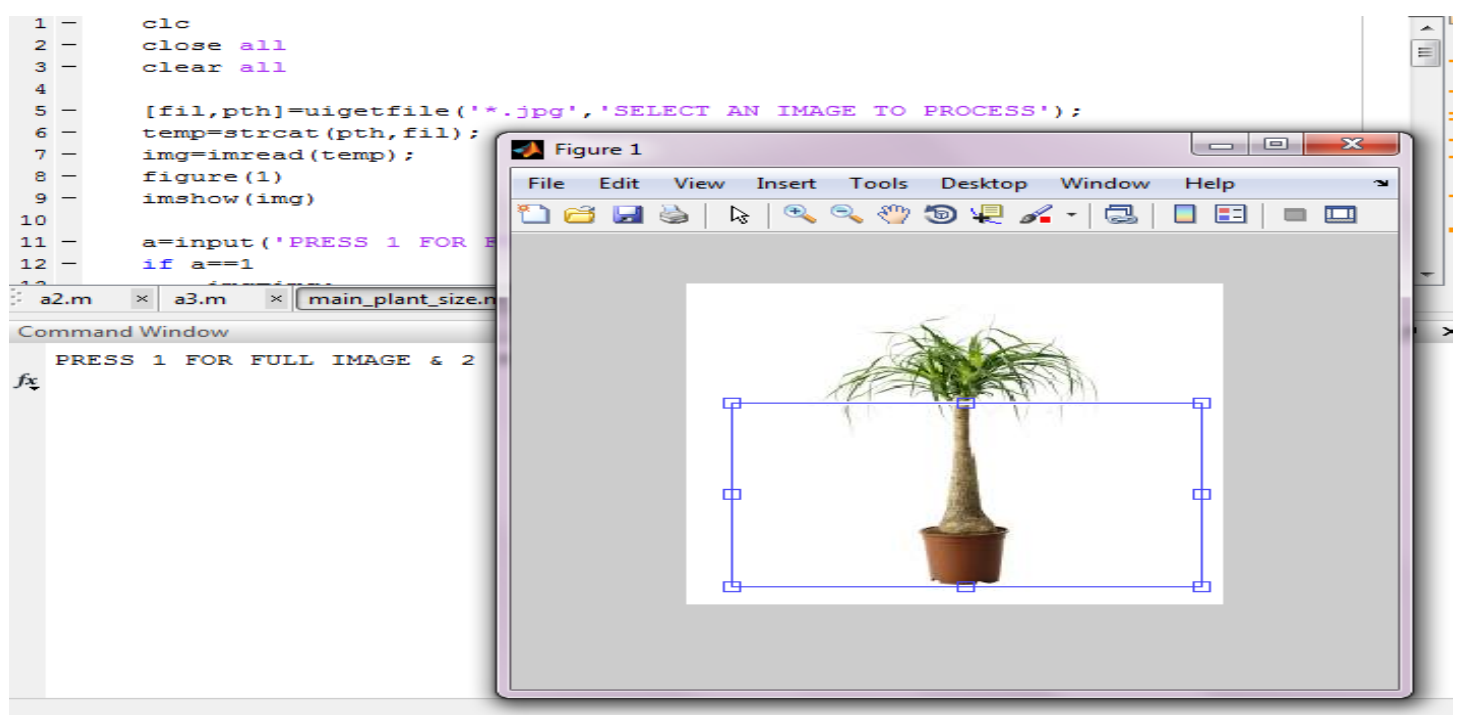

Figure 4. Selection of cropped image

Figure 5 is presenting the plant image with highlighted edges as well as the measured values of height, maximum and minimum width of a plant in terms of pixels.

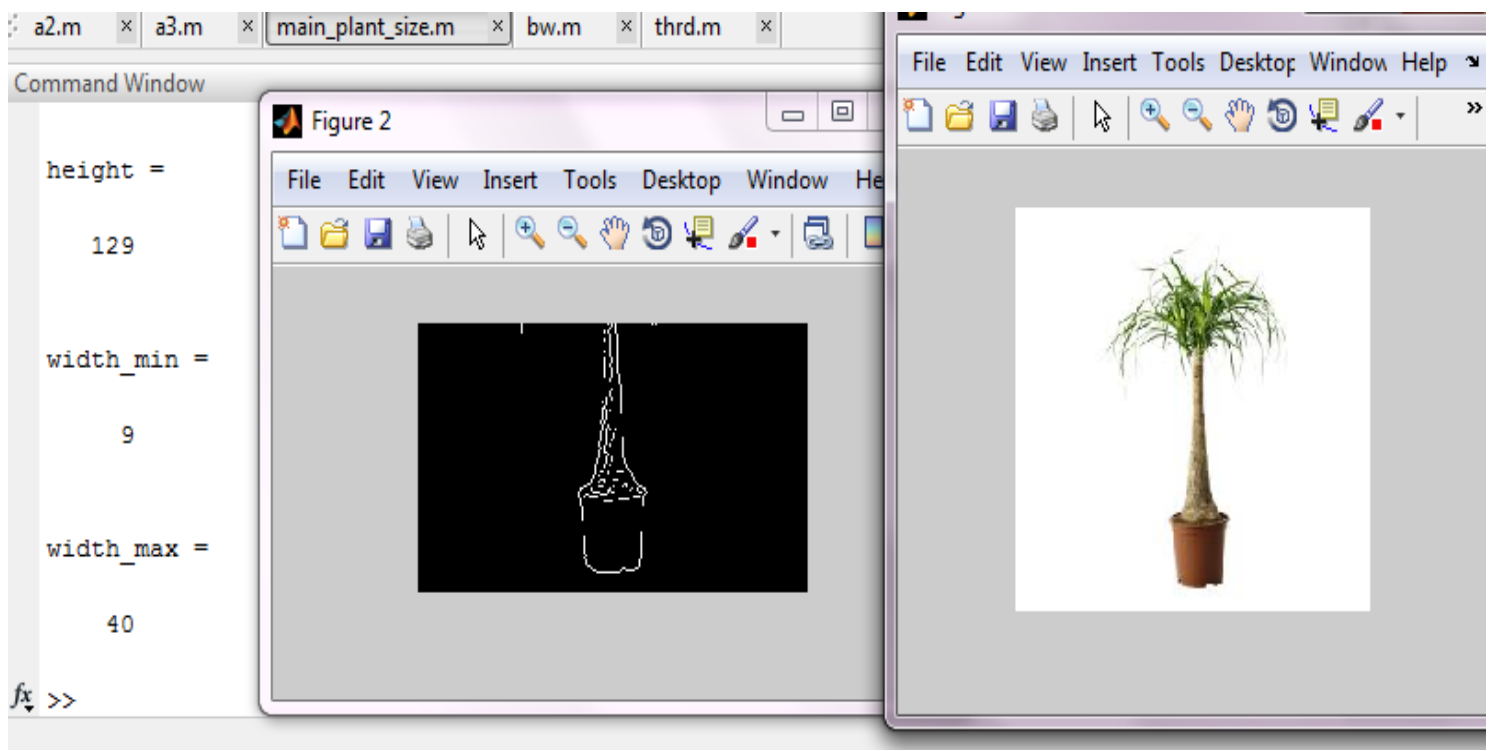

Figure 5. Edge detected image

Figure 6 is presenting the full plant image with highlighted edges as well as the measured values of height and width (minimum and maximum) of a plant in terms of pixels. 
International Journal of Computer Science, Engineering and Applications (IJCSEA) Vol.3, No.5, October 2013

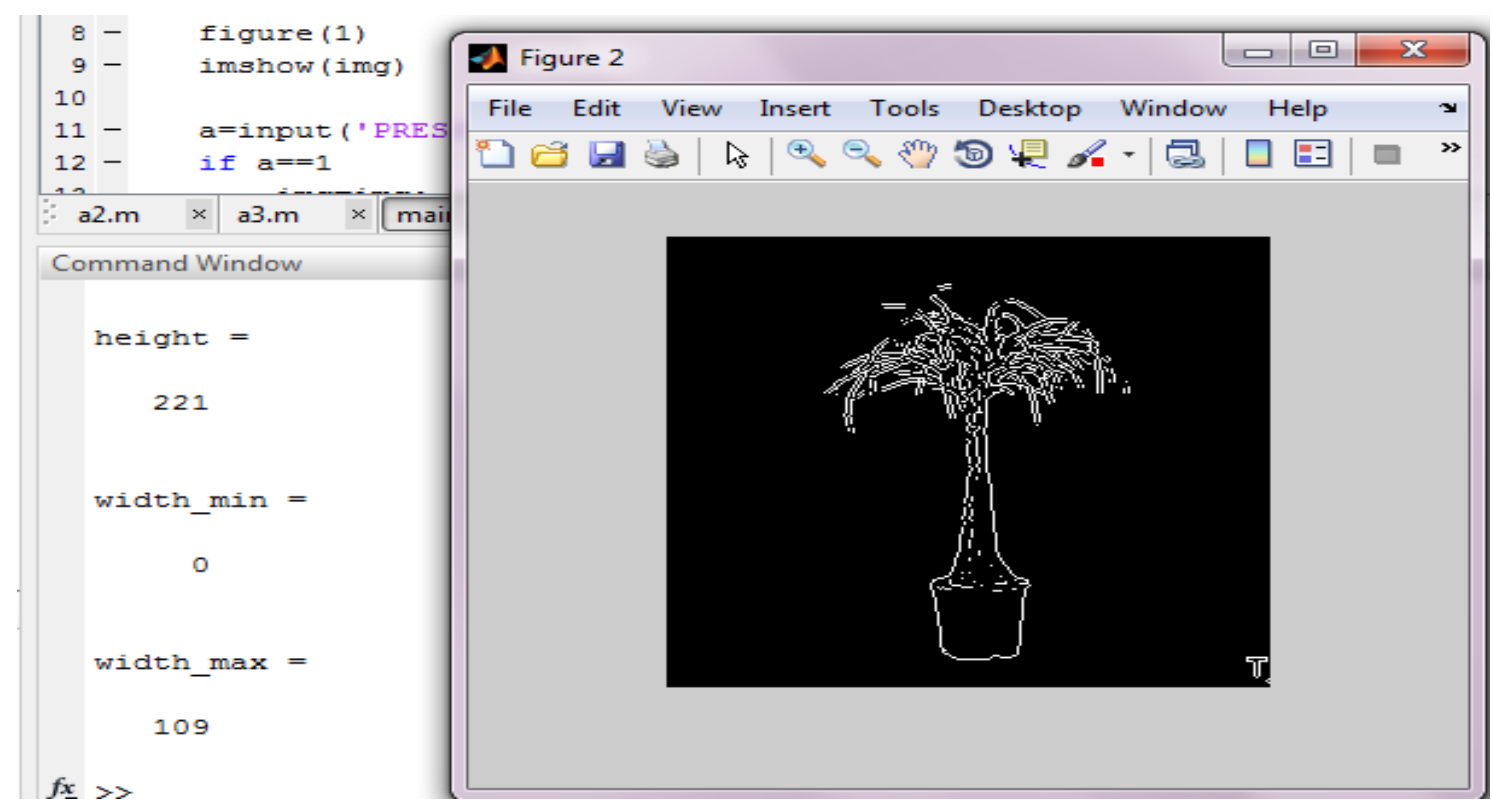

Figure 6. Edge detected for full plant's image

Table 1. Testing of the plant at different days

\begin{tabular}{|c|c|c|c|}
\hline Days & Height in pixels & $\begin{array}{c}\text { Minimum width in } \\
\text { pixels }\end{array}$ & $\begin{array}{c}\text { Maximum width in } \\
\text { pixels }\end{array}$ \\
\hline Day 1 & 129 & 9 & 40 \\
\hline Day 5 & 134 & 11 & 42 \\
\hline Day 12 & 241 & 15 & 46 \\
\hline Day 22 & 250 & 20 & 51 \\
\hline
\end{tabular}

\section{CONCLUSION}

In this research, growth of a plant is measured using interface between digital camera and a computer. This interface allows a user to get continuous video of the plants. All the proposed tasks of the thesis were done. The servo motor is added to the system to get panoramic images. A MATLAB GUI has been developed for performing sobel edge detection on a plant image to determine its height and width. Two different types of options for the selection of a plant image are provided. Either the full image or cropping of the image can be done. The algorithm provides height and maximum and minimum width of the plant's stem in terms of pixels. 


\section{REFERENCES}

[1] N.M.Khairi, M.A.Marni Shah Rizam, M.S.B. Tahir, N.M. Naimah, M.I. Zainol Abidin,"Optimization of strain gauge for stem measurement using PIC based instrumentation," System Engineering and Technology (ICSET), 2011 IEEE International Conference, pp.196-199, 27-28 June 2011.

[2] Jiaxing Che, Chunjiang Zhao, Yunhe Zhang, Cheng Wang, Xiaojun Qiao, Xinlu Zhang, "Plant stem diameter measuring device based on computer vision and embedded system," World Automation Congress (WAC), 2010, pp.51-55, 19-23 Sept. 2010.

[3] Ariff, E.A.R.E. Suratman, M.N.Abdullah," Stomatal conductance, chlorophyll content, diameter and heightin different growth stages of rubber tree (Hevea brasiliensis) saplings," Business, Engineering and Industrial Applications (ISBEIA), 2011 IEEE Symposium, pp.84-88, 25-28 Sept. 2011.

[4] Nick Kanopoulos, et.al. ; "Design of an Image Edge Detection Filter using the Sobel Operator", Journal of Solid State Circuits, IEEE, vol. 23, Issue: 2, pp. 358-367, April 1988.

[5] Beant Kaur, Mr. Anil Garg, "Comparative study of different edge detection techniques" International Journal of Engineering Science and Technology (IJEST) Vol. 3 No. 3 March 2011.

[6] Wenshuo Gao; Xiaoguang Zhang; Lei Yang; Huizhong Liu, "An improved Sobel edge detection," Computer Science and Information Technology (ICCSIT), 2010 3rd IEEE International Conference on , vol.5, pp.67,71, 9-11 July 2010.

[7] Chunxi Ma, et al.; "An improved Sobel algorithm based on median filter", Institute of Electrical and Electronics Engineers, 2nd International IEEE conference, China, Volume: 1, pp. 88-93, Aug 1, 2010.

[8] Klepper B, Browning VD, Taylor HM. "Stem diameter in relation to plant water status", PlantPhysiology, 1971(48) 683-685.

[9] S.O.Link, M.E.Thiede and M.G.van Bavel, "An improved strain-gauge device for continuous field measurement of stem and fruit diameter," Journal of Experimental Botony, Vol.49, No. 326, pp. 1583- 1587. September 1998.

[10] Mcburney T, Costigan PA. "The relationship between stem diameter and water potentials in stems of young cabbage plants", Journal of Experimental Botany, 1984(35)1787-1793.

[11] C.Y. Liao, V.V. Podrazsky, and G. B. Liu, "Diameter and height growth analysis for individual white pine trees in the area of Kostelec nad Cernymilesy," Journal of Forest Science, vol. 49, pp. 544-551, 2003.

[12] T. Kärki, M. Maltamo, and K. Eerikäinen, "Diameter distribution, stem volume and stem quality models for grey alder (Alnus incana) in eastern Finland," New Forests, vol. 20, pp. 65-86, 2000.

[13] X.H. Sang, Y.Y. Wu, Y.G. Zhao, and M.Y. Xu, "Comparison of growth of four types of Atractylodes Lancea (Thunb.) DC," Journal of Anhui Agricultural Sciences, vol. 36, pp. 7726 - 7727, 2008.

\section{Authors Biography}

Balwinder Singh has obtained his Bachelor of Technology degree from National Institute of Technology, Jalandhar and Master ofTechnology degree from University Centre for Inst. \& Microelectronics(UCIM), Punjab University, Chandigah in 2002 and 2004 respectively. He is currently serving as Senior Engineer ACS division in Centre for Development ofAdvanced Computing (CDAC), Mohali and is a part of the teachingfaculty and also pursuing Phd from GNDU Amritsar. He has 8+ years of teaching experience to both undergraduate and postgraduate students. He has published

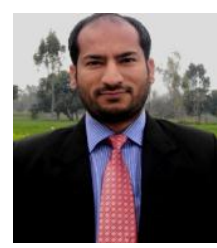
three books and many papers in the International \& National Journal and Conferences. His current interest includes Genetic algorithms, Low Power techniques, VLSI Design \& Testing, and System on Chip.

Ramandeep Kaur has done her bachelor of technology degree in Electronics and Communication Engineering with first division from Punjab Technical University Jalandhar in year 2010 and currently pursuing her Master of Technology degree in Embedded System from CDAC, Mohali. Her areas of interests are microcontrollers, Analog and Digital electronics and communications.

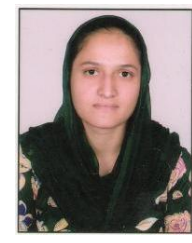

